\title{
ITTALKS: A Case Study in the Semantic Web and DAML
}

\author{
R. Scott Cost, Tim Finin, Anupam Joshi, Yun Peng, Charles Nicholas, Harry Chen, \\ Lalana Kagal, Filip Perich, Youyong Zou, Sovrin Tolia
}

\author{
Laboratory for Advanced Information Technology \\ University of Maryland Baltimore County
}

\begin{abstract}
Effective use of the vast quantity of information now available on the web will require the use of "semantic web" markup languages such as the DARPA Agents Markup Language (DAML). Such languages will enable the automated gathering and processing of much information that is currently available but insufficiently utilized. As part of our exploration of semantic web technology, and DAML in particular, we have constructed ITTALKS, a web-based system for automatic and intelligent notification of information technology talks. In this paper, we describe the ITTALKS system, and discuss the numerous ways in which the use of semantic web concepts and DAML extend its ability to provide an intelligent online service to both the human community and the agents assisting them.
\end{abstract}

\section{Introduction $^{1}$}

With the vast quantity of information now available on the Internet, there is a need to manage this information by marking it up with a semantic language, such as DARPA Agent Markup Language (DAML) [DAML], and using intelligent search engines, in conjunction with ontologybased matching, to provide better search results. The aim of the semantic web is to make the web more machinereadable, in order to allow intelligent agents to retrieve and manipulate pertinent information. The key goal of the DAML program is to develop a semantic web markup language that is sufficiently rich to support intelligent agents and other applications. Today's agents are not tightly integrated into the web infrastructure. If our goal is to have agents acting upon and conversing about web objects, they will have to be seamlessly integrated with the web, and take advantage of existing infrastructure whenever possible (e.g., message sending, security, authentication, directory services, and application service

\footnotetext{
${ }^{1}$ This work was supported in part by the Defense Advanced Research Projects Agency under contract F30602-00-2-0 591 AO K528 as part of the DAML program (http://daml.org/). Corresponding author: R. Scott Cost, mailto:cost@cs.umbc.edu, Computer Science and Electrical Engineering, University of Maryland Baltimore County, Baltimore MD 21250. Draft of May 17, 2001 submitted to the International Semantic Web Workshop (SWWS) -- Infrastructure and Applications for the Semantic Web, July 30 - 31, 2001, Stanford University.
}

frameworks). We believe that DAML will be central to the realization of this goal.

In support of this claim, we have constructed a real, fielded application, ITTALKS, which supports user and agent interaction in the domain of talk discovery. It also provides a simple web-driven infrastructure for interacting agents. In addition, ITTALKS serves as a platform for designing and prototyping the software components required to enable developers to create intelligent software agents capable of understanding and processing information and knowledge encoded in DAML and other semantically rich markup languages. To date, we have focused on developing the support and infrastructure required for intelligent agents to integrate into an environment of web browsers, servers, application server platforms, and associated supporting languages (e.g., WEB/SQL, WEBL), protocols (e.g., SSL, S/MIME, WAP, eSpeak), services (e.g., LDAP) and underlying technologies (e.g., Java, Jini, PKI).

On the surface, ITTALKS is a web portal offering access to information about talks, seminars and colloquia related to information technology (IT). It is organized around domains, which typically represent event hosting organizations such as universities, research laboratories or professional groups, and which are represented by independent web sites. ITTALKS utilizes DAML for its knowledge base representation, reasoning, and agent communication. DAML is used to markup all the information in the knowledge base to provide additional reasoning capabilities otherwise unavailable. With information stored in a semantically machine understandable format, the computer can deduce information, which would otherwise be impossible in a standard database system. For example, if both ITTALKS and the user agree on a common semantics, the ITTALKS web portal can provide not only the talks that correspond to the user's profile in terms of interest, time, and location constraints, but can further filter the IT events based on information about the user's personal schedule, inferred location at the time of the talk, distance and current traffic patterns, etc. ITTALKS can also dynamically update the user's profile with incremental learning of the user's usage patterns. 


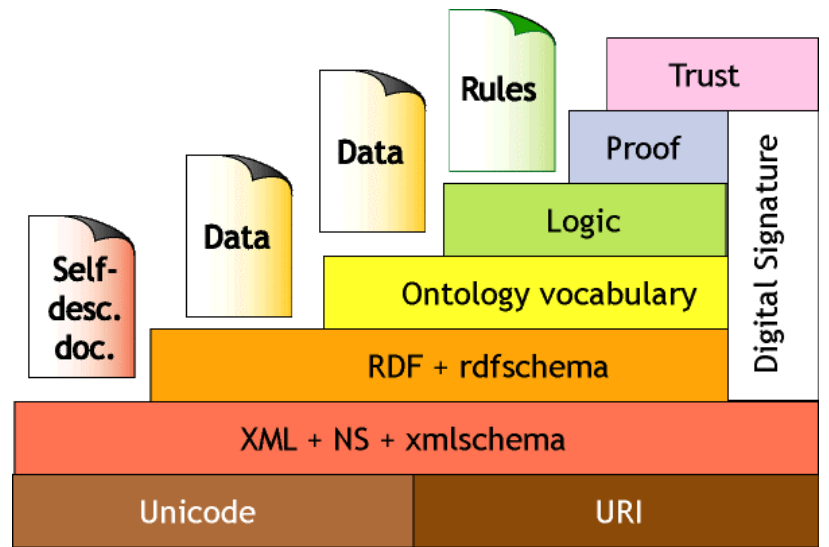

Figure 1: Tim Berners Lee's vision of the Semantic Web is founded on a base that includes URIs, XML and RDF.

ITTALKS demonstrates the power of markup languages such as DAML for the semantic web, drawing on its ability to represent ontologies, agent content languages and its ability to improve the functionality of agents on the web. We have developed DAML-encoded ontologies for describing event, temporal, spatial, personal, and conversational information, which enable us to represent all required knowledge in a DAML-encoded format, and which also enable us to execute a computer understandable conversation. In addition, we have created several DAMLencoded classification ontologies, which provide us with additional reasoning capabilities in order to find the best matching IT talks for a particular user. By combining the features of current existing web applications with the DAML-based knowledge and reasoning capabilities, ITTALKS presents a true semantic web application.

\section{Background}

The semantic web [Berners-Lee et al., '01] is a vision in which web pages are augmented with information and data that is expressed in a way that facilitates its understanding my machines. The current human-centered web is still largely encoded in HTML, which focuses largely on how text and images would be rendered for human viewing. Over the past few years we have seen a rapid increase in he use of XML as an alternative encoding, one that is intended primarily for machine processing. The machine which process XML documents can be the end consumers of the information or they can be used to transform the information into a form appropriate for human understanding (e.g., as HTML, graphics, synthesized speech, etc.) As a representation language, XML provides essentially a mechanism to declare and use simple data structures and thus leave much to be desired as a language in which to express complex knowledge. Recent enhancements to basic XML, such as XML Scheme, address some of the shortcomings, but still do not result in an adequate language for representing and reasoning about the kind of knowledge essential to realizing the semantic web vision.

RDF (Resource Description Framework) [RDF] and RDFS (RDF Schema) [RDFS] attempt to address these deficiencies by building on top of XML. They provide representation frameworks that are roughly the equivalent to semantic networks in the case of RDF and very simple frame languages in the case of RDFS. However, RDFS is still quite limited as a knowledge representation language, lacking support for variables, general quantification, rules, etc. DAML is one attempt to build on XML, RDF and RDFS and produce a language that is well suited for building the semantic web.

The goal of the DAML program (http://www.daml.org/), which officially began in August 2000, is to develop a universal semantic web markup language that is sufficiently rich to support intelligent agents and other applications. DAML can dramatically improve traditional ad hoc information retrieval because its semantics will improve the quality of retrieval results. Also, it will allow the intelligent agents of tomorrow to retrieve and manipulate the information on the semantic web.

\section{ITTALKS}

As part of UMBC's role in the DAML Program, we have developed ITTALKS; a web portal that offers access to information about talks, seminars, colloquia, and other information technology (IT) related events. ITTALKS provides users with numerous details describing the IT events, including location, speaker, hosting organization, and talk topic. Below, we discuss various aspects of the system in more detail.

Unlike other web services, ITTALKS employs DAML for knowledge base representation, reasoning, and agent communication. The use of DAML to represent information in its knowledge base enables more sophisticated reasoning than would otherwise be available. For example, a simpler representation scheme might be able to provide the user with talks based on interest, time and location. When both ITTALKS and the user agree on a common semantics, the ITTALKS web portal will be able to perform further filtering, based on sophisticated inference. In addition to enhancing knowledge representation and reasoning, DAML is used for all communication, including simple messages and queries, using the ITTALKS defined ontology. Moreover, ITTALKS offers the capability for each user to use his/her personal agent to communicate with ITTALKS on his/her behalf and provide a higher level of service. 


\section{Users}

ITTALKS can be used anonymously, or, more effectively, with personalized user accounts. Users have the option to register with ITTALKS either by entering information manually via web forms, or by providing the location (URL) of a universally accessible DAMLized personal profile, which includes information such as the users location, his/her interests and contact details, as well as a schedule. This schedule might be as rudimentary as a list of available time periods for given days, or could even include a detailed schedule for each day. Subsequently, this information is used to provide each user with a personalized view of the site, displaying only talks that match the user's interests and/or schedule.

Since DAML is not yet in widespread use, ITTALKS provides a tool for creating a DAML personal profile. Currently, the tool constructs a profile containing only items used by the ITTALKS system. However, we believe that the profile, in one form or another, will ultimately provide a unique and universal point for obtaining personal information about the user, not just for ITTALKS, but for all information needs, and will include any sort of information the user would like to share. In the future, all services that require personal information about the user should access the same user profile, eliminating the need for the user to repeatedly enter the same information for a multitude of services. We believe that the new standard for XML Signature and Encryption under development may provide a mechanism by which users can have some control over access to parts of their profile.

\section{Domains}

To support our vision of a universal resource for the international IT research community, ITTALKS is organized around domains, which typically represent event hosting organization such as universities, research laboratories or professional groups. Each domain is represented by a separate web site and is independently maintained by a moderator who can, among other things, define the scope of the domain and delegate to other registered users the ability to edit talk entries. For example, the stanford.ittalks.org domain might be configured to include only talks hosted at Stanford University. On the other hand, another domain, sri.ittalks.org, might be configured to include not only talks about semantic web topics that are held at SRI, but also those at Stanford, as well as any talks within 15 mile range of the SRI facility in Palo Alto.

\section{Access}

The ITTALKS system is accessible either to users directly via the web, or to agents acting on their behalf. The web portal provides numerous features, including registration, search, entry and domain administration. An agent-based interface allows interaction with user agents or other services.

Human Interface. The web portal allows a user to browse desired information in a variety of formats, to provide the highest degree of interoperability. It permits a user to retrieve information in DAML, standard HTML format, which includes a short DAML annotation for DAMLenabled web crawlers, or WML [WML] format, which supports WAP enabled phones. The ITTALKS web portal also has the ability to generate RDF Site Summary (RSS) [RSS] files for certain queries. These RSS files can then be used for various external purposes, such as displaying upcoming talks on a departmental web site for some particular university or domain.

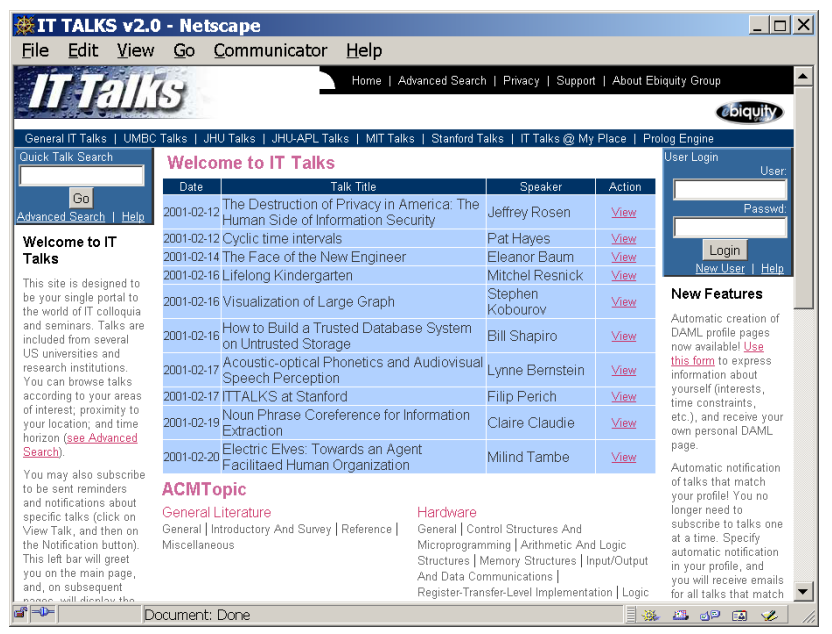

Figure 2: A screenshot depicting the main page of the ITTALKS system.

Agent Interface. To provide access for agent based services, ITTALKS makes use of Jackal [Jackal], a communication infrastructure for Java-based agents developed by our research group at UMBC. Jackal is a Java package, which provides a comprehensive communications infrastructure while maintaining maximum flexibility and ease of integration. The heart of Jackal is a simple conversation system, serving to maintain context for concurrent threads of conversation while providing a guide for judging behavioral correctness and modeling the actions of other agents. Jackal provides facilities for creating and manipulating user-defined conversation structures of arbitrary extent. Jackal has a very modular, loosely coupled architecture, designed to support maximal concurrency among components, accomplished with the use of multiple threads and buffered interfaces between subsystems. Its concise API allows for comprehensive specification of message requests, and for blocking or nonblocking message retrieval. Currently, it facilitates the use of KQML agent communication language and employs a sophisticated protocol for agent naming, addressing and identity (KNS); however, it is in the process of adapting to the FIPA standards. In addition, our research group, in 


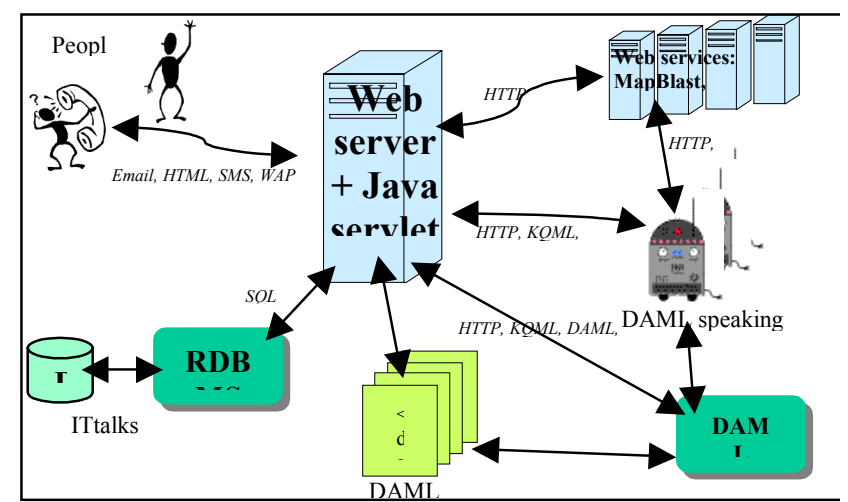

Figure 3: The architecture for ITTALKS is built around a web server backed by a relational database. Interfaces are provided to human users, software agents andweb services.

cooperation with other universities, is developing a DAML ontology for the necessary conversation protocols.

\section{Agents}

In order to extend the capabilities of the system, we have defined a number of agents that support the operation of ITTALKS. Some can be seen as supporting services (such as external information services), while others we assume will exist in the general environment in the future.

ITTALKS Agent. The ITTALKS agent is a front-end for the ITTALKS system. It interacts with ITTALKS through the same web-based interface as human users, but communicates via an ACL with other agents on the web, extending the system's accessibility. At present, the agent does not support any advanced functionality, but acts primarily as a gateway for agent access.

User Agents. One longtime goal of agent research is that users will be represented online by agents that can service queries and filter information for them. While ITTALKS does not require that such agents exist, we recognize the added power that could be gained by the use of such agents. Therefore, ITTALKS supports interaction with User Agents as well as their human counterparts. The User Agent that we have developed understands DAML, supports sophisticated reasoning, and communicates via a standard agent communication language. Reasoning is accomplished with the use of the XSB, a logic programming and deductive database system for Unix and Windows developed at SUNY Stony Brook.

Classifier Agent. ITTALKS uses a Classifier (or recommender) Agent that is invoked when a user is entering a new talk. Based on the talk's abstract, the Classifier returns ACM Classification Hierarchy Classification numbers along with a rank, in descending order. Using a local table of classification numbers and names, ITTALKS suggests to the user ten possible topics.

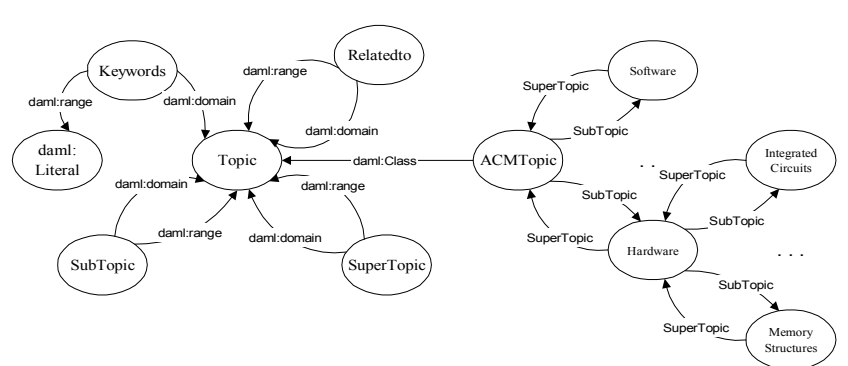

Figure 4: The Ontologies used by IITALKS are relatively simple, such as the topics ontology used to describe talk topics and user interests.

MapQuest $^{\mathrm{TM}}$ Agent. The MapQuest $^{\mathrm{TM}}$ Agent is a wrapper agent that allows ITTALKS to make use of external services. It interacts directly with agents (e.g. the ITTALKS agent, User Agents), and accepts requests for information such as the distance between two known locations. It then phrases an appropriate request to the MapQuest $^{\mathrm{TM}}$ system [MapQuest], parses the results, and generates an appropriate response. Note that this agent could be generically named a Distance Agent, and make use of any external service (or combination of several, as needed).

\section{Ontologies}

The ITTALKS system is based on a set of Ontologies (http://daml.umbc.edu/ontologies) that are used to describe talks and the things associated with them, e.g., people, places, topics and interests, schedules, etc. Figure 5 shows some of the dependencies that exist among these ontologies. The ontologies are used in the representation and processing of DAML descriptions and also as "conceptual schemata" against which the database and various software APIs are built.

We have developed a general ontology for describing the topics of arbitrary talks and papers. Using this, we have implemented an ontology to describe IT related talks based on the ACM's Computer Classification System. In addition, we currently are developing a DAML ontology for IT talks based on a portion of the Open Directory, and are considering additional classification ontologies. Figure 4 sketches some of the major classes and properties in these ontologies. These topic ontologies are used to describe talks as well as the users' interests throughout the system. This includes an automated talk classification, for which we have obtained a training collection for the ACM CCS and are also generating an Open Directory training collection to develop the necessary components. In addition, the DAML ontologies will give a user the ability to add additional assertions in DAML to further characterize their interests. Lastly, we are also in the process of developing a component that can map topics in one ontology into topics in another, by taking advantage of the fact that nodes in each ontology have an associated collection of text as well as DAML information. 


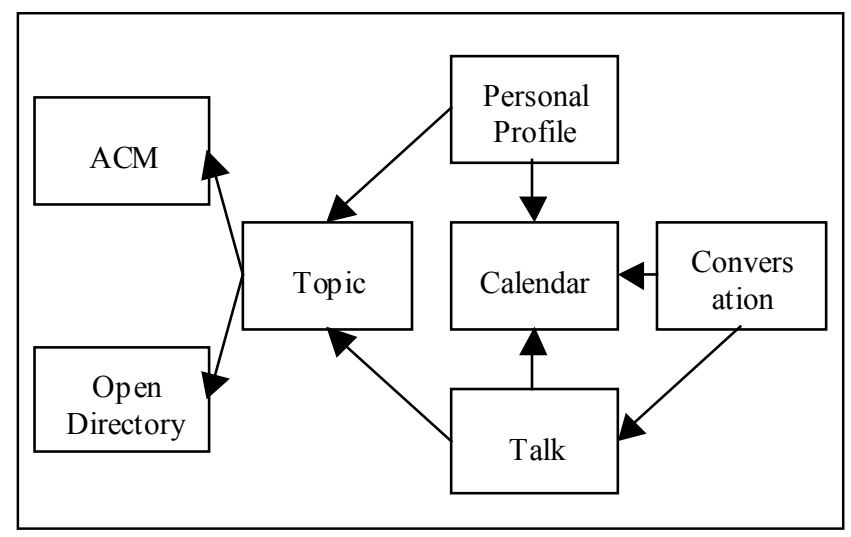

\section{Data Entry}

Currently ITTALKS requires that information about talks to be manually entered via a web form interface, or be available in a DAML description available at a given URL. Although we have made this as simple as possible (e.g., by supporting automatic form completion using information from the knowledge base and user's DAML profile) it is still a time consuming process. Therefore, we are developing a focused web spider to collect talk announcements from open sources on the web. This spider will identify key information items in these announcements using a text extraction system, and will automatically add information to the ITTALKS knowledge base. We are working with the Lockheed-Martin research group on the above task, and will use their AeroText information extraction system [AeroText].

\section{Architecture}

The current implementation of ITTALKS uses a relational database, in combination with a web server to provide user access to the system. To enable agents to access the system, the ITTALKS provides an interface for agentbased communication.

Database. The main software packages that are used in the ITTALKS system are the MySQL relational database software and a combination of Apache and Tomcat as the web portal servers. The contents of the ITTALKS knowledge base are stored in a database whose schema is closely mapped to our ontologies describing events, people, topics and locations. We have chosen MySQL because of its known reliability, and because we required software with a license that allows us to make the ITTALKS package available to additional academic and commercial institutions.

Web Server. As stated above, for our web, we have chosen a combination of Apache and Tomcat. This enables us to present the IT talk descriptions to the user using Java servlets and JSP files, which dynamically generate requested information in DAML, XML, HTML, RSS, and WML formats. The current ITTALKS implementation can provide information suitable for viewing on either a standard, computer-based or a WAP-enabled cellular phone.

Extensions. In addition, we are currently employing the Jackal agent communication infrastructure developed at UMBC and the Lockheed-Martin's AeroText information extraction system in order to facilitate ITTALKS-user agent interaction and the automated text extraction, respectively. We are in the process of modifying Jackal to provide support for FIPA ACL interoperability. Also, we are considering the possible replacement of MySQL with native XML database software such as dbXML.

\section{Scenarios}

We describe here a couple of typical interactions that illustrate some of the features of ITTALKS. The first involves direct use by a human user, and the second, advanced features provided through the use of agents.

\section{Human Interaction}

In this first scenario, a user, Jim, learns from his colleagues about the existence of the ITTALKS web portal as a source of IT related events in his area; Jim is affiliated with Stanford University.

Jim directs his browser to the www.ittalks.org main page. Seeing a link to stanford.ittalks.org (a Stanford ITTALKS domain), he selects it, and is presented with a new page listing upcoming talks that are scheduled at Stanford, SRI and other locations within a 15-mile radius (the default distance for the Stanford domain).

Jim browses the web site, viewing announcements for various talks matching his interests and preferred locations (as provided in his explicit search queries). $\mathrm{He}$ is impressed that he can see the talk information not only in HTML, but also in DAML, RSS and WML formats. Finding a talk of potential interest to a colleague, Jim takes advantage of the invitation feature, which allows him to send an invitational e-mail to any of his friends for any of the listed talks. Finally, using the personalize link on the bottom of the page, Jim creates his own ittalks.org main page, by providing the URL of his DAML-encoded profile. This customized page, listing talks based on his preferences, will be Jim's entrance to the ITTALKS site whenever her returns.

\section{Agent Interaction}

This scenario assumes that user Jim has already registered with ITTALKS, and has left instructions with the system to be notified of the occurrence of certain types of talks. 


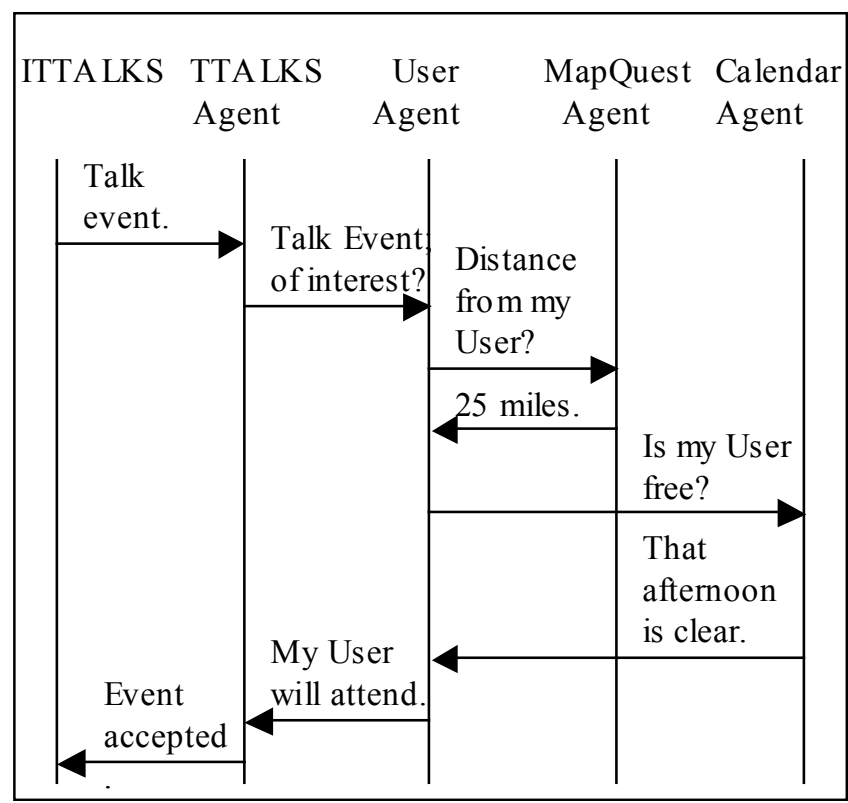

In the course of operation, ITTALKS discovers that there is an upcoming talk that may interest Jim, and of which Jim has not been notified. Based on information in Jim's preferences, which have been obtained from his online, DAML-encoded profile and from information entered directly, ITTALKS opts to notify Jim's User Agent directly. This is done via ITTALKS own agent, which forwards the message using an ACL.

Upon receiving this information, Jim's User Agent needs to know more; it consults with Jim's Calendar agent to determine his availability, and with the MapQuest ${ }^{\mathrm{TM}}$ agent to find the distance from Jim's predicted location at the time of the talk. Some more sophisticated interactions might take place at this time; for example, the Calendar and User agents may decide to alter Jim's schedule, and proceed to contact the User agent of some other individual. After making a decision, the User Agent will send a notification back to the ITTALKS agent indicating that Jim will/will not plan to attend. The ITTALKS agent will make the appropriate adjustments at the ITTALKS site.

\section{Benefits of DAML}

We believe that ITTALKS benefits significantly from its use of a semantic markup language such as DAML. DAML is used to specify ontologies that we use extensively in our system. It is also used for personal profiles, and as an agent content language. Without DAML, specifying schedules, interests and assertions about topics would be very difficult. In ITTALKS, a user can specify that according to the user a couple of topics are equivalent or related or dissimilar etc. This will allow ITTALKS to tailor the searching of talks to the users needs. As an agent content language, DAML provides more flexible semantics than KIF or other content languages that only provided syntax.

\section{Interoperability Standard}

As an interoperability layer, DAML allows the content of ITTALKS to be easily shared with other applications and agents. For example, a Centaurus room manager agent [Kagal et al., '01] could watch ITTALKS for events happening in a room for which it is responsible in order to enable better scheduling. DAML also acts as an interoperability standard allowing other sites to make their talks available for inclusion in ITTALKS by publishing announcements marked up in our ontology.

\section{Data Entry Support}

ITTALKS supports intelligent form filling, making it easier for users to enter and edit information in their profiles, and also to enter and edit talk announcements and other basic information. In addition, we provide automatic form filling when an editor tries to enter information about an entity (e.g. a talk, person, room) that already present in the knowledge base.

Entering Talks. In order to make ITTALKS successful, as we need to make it as easy as possible new talk descriptions to be entered into the system. We are addressing this problem using three complimentary approaches: an enhanced web interface, accepting marked up announcements, and automated text extraction. DAML plays a key role in the first two and is the target representation for the third.

Enhancing the Web Interface. We've used several techniques to enhance the web form interface for entering talk announcements. One of the simplest and most effective is to recognize then some of the information being entered about an object such as a person, a room or an organization has already been entered into the ITTALKS system and to "pre-fill" the remaining parts of the form from our stored information. For example, most talks at an organization are given in a small number of rooms. Once the complete information about a particular room (e.g., room number, building, address, seating capacity, longitude and latitude, $\mathrm{A} / \mathrm{V}$ equipment, networking connection, etc.) has been entered for one talk, it need not be entered again.

Although the current implementation of this does not directly use DAML, its use can support a more generalized version of a web form-filling assistant. The approach depends on two ideas: (i) tagging web form widgets with DAML descriptions of what they represent and (ii) capturing dependencies among data items in DAML and (iii) compiling these dependencies into an appropriate execution form (e.g., Javascript procedures) that can drive the web form interface. 
Text Classification. In order for ITTALKS to filter talk announcements on topic matches, we need to know the appropriate topics for each talk. Initially, we required that users manually select appropriate topic categories from a web interface to the ACM CCS hierarchy. This turns out to be a daunting task requiring the user to navigate in a hierarchy of nearly 300 topics, many of which about whose meaning he will not be sure. Some users will face a similar problem in trying to select topics to characterize their own interests. Ultimately we would like to use more that one topic hierarchy to classify both talk topics and user interests (e.g., ACM CCS and Open Directory nodes), which makes the problem even more difficult for our users.

To address this problem, we have built an automatic text classifier that can suggest terms in a hierarchy that are appropriate for classifying a talk based on its title and abstract. The classifier package used was from the Bag Of Words (BOW) [McCallum et al., '96] toolkit by Andrew McCallum at CMU. This library provides support for a wide variety of text classification and retrieval algorithms. We used the Naive Bayes algorithm, which is widely used in the classification literature, fairly effective, and quick to learn the 285 classes in our test collection. We plan to use the same classification agent to suggest interest terms for users based on the text found by searching their web pages.

Accepting Marked Up Announcements. One of the simplest ways to enter new talk announcements is to provide them as a document that is already marked up. The current ITTALKS interface allows one to enter a URL for a talk announcement that is assumed to be marked up in ontologies that ITTALKS understands. Currently, these are just the "native" ontologies that we have built for this application. In general, if some talk announcements were available with semantic markup using other ontologies, it might be possible to provide rules and transformation that could map or partially map the information into the ITTALKS ontologies. We expect that, as the semantic web develops, it will be more and more likely that talk announcements with some meaningful mark up will be found on the web.

Automated Information Extraction from Text. We would like to be able to process talk announcements in plain text or HTML and automatically identify and extract the key information required by ITTALKS. This would allow us to fill the ITTALKS database with information obtained from announcements delivered via email lists or found on the web. The problem of recognizing and extracting information from talk announcements has been studied before [Eliassi-Rad 2000, Ciravegna 2000] mostly in the context of using it as a machine learning application. We are developing a information extraction use the Aerotext [Childs 2001, Aerotext] system that can identify and extract the information found in a typical talk announcement and use this to automatically produce a version marked up in DAML which can then be entered in the ITTALKS database.

\section{User Profiles}

We use personal profiles to help ITTALKS meet the requirements of individual users. A profile is a widely accessible source of information about the user, marked DAML, to which other services and individuals can refer. In the future, such a profile may be used by all web-based services that the user wants to access. The profile will ultimately provide a unique and universal point for obtaining personal information about the user for all services, preventing the need for duplication and potential inconsistencies. This profile can be easily shared, and with the use of DAML, will allow more expressive content for schedules, preferences and interests. The notion of a personal profile and a user agent are closely linked; a user might have one or the other, or both. The profile would likely express much of the information that might be encoded in a user agent's knowledge base. Conversely, an agent would likely be able to answer queries about information contained in a profile.

\section{Distributed Trust and Belief}

Agents face a difficult problem of knowing what information sources (e.g. documents, web pages, agents) to believe and trust in an open, distributed and dynamic world, and how to integrate and fuse potentially contradictory information. DAML can be used to support distributed trust and reputation management. This will form the basis of a logic for distributed belief transfer that will enable more sophisticated, semantically-driven rulebased techniques for information integration and fusion.

We are making use of DAML's expressiveness and employing it to describe security policies, credentials and trust relationships, which form the basis of trust management. These policies contain more semantic meaning, allowing different policies to be integrated and conflicts to be resolved relatively easily. Also, it will be possible for other applications to interpret the agent's credentials, e.g. authorization certificates, correctly, making these credentials universal.

Similarly, describing beliefs and associating levels of trust with these beliefs is more straightforward and the deduction of belief is uniform by different applications and services.

\section{Modularity}

With the use of DAML, we can define several ontologies for topics and switch between them with ease. Furthermore, to restrict the retrieval results, a user can perform the search with respect to a certain set of ontologies, such as the ACM or Open Directory Classification. 


\section{Agent Communication Language}

DAML and ACLs can be successfully integrated. DAML documents will be the objects of discourse for agents that will create, access, modify, enrich and manage DAML documents as a way to disseminate and share knowledge. Agents will need to communicate with one another not only to exchange DAML documents but also to exchange informational attitudes about DAML documents. Using an Agent Communication Languages (ACL) agents can "talk" about DAML documents. Integrating ACL work and concepts with a universe of DAML content is our first goal. Using DAML as an agent content language will add more meaning to the message.

\section{Current Status/Observations}

We have currently implemented the website and display normal HTML with embedded DAML. There is an option of viewing only DAML content for a certain page or a specific talk or specific user. All these requests are via HTTP. We also provide a form-based interface to add/modify the database including talks and users. We have a two level moderation, with the root being the highest. The root can delegate rights to certain users making them editors and allowing them to edit a particular domain. We also offer tools to generate personal profiles. We allow users to filter talks by interest and location. We also have a MapQuest ${ }^{\mathrm{TM}}$ agent that calculates the distance between the user's location and the talk.

\section{Future Directions}

Since most users do not currently have personal agents, we have been developing one that can be used with this system. It is our goal, however, that ITTALKS will be able to interact with external agents of any type. The agent we are developing reasons about the users interests, schedules, assertions and that uses the MapQuest ${ }^{\mathrm{TM}}$ agent to figure out if the user will be able to attend an interesting talk on a certain date.

We are developing a framework to use DAML in distributed trust and belief. DAML expressions on a web page that encodes a statement or other speech act by an agent are signed to provide authentication and integrity. We are working on an ontology to describe permissions, obligations and policies in DAML and allow agents to make statements about them and delegate them.

Currently we use only HTTP, but plan to move to Jackal for agent communication. Jackal currently supports only KQML and we are in the process of adapting it to the FIPA standards. In addition, our research group, in cooperation with other universities, is developing a DAML ontology for the necessary conversation protocols.
In order to make the process of data entry more efficient, we are developing a focused web spider, which will collect talk announcements from open source on the web and to identify the key information in these announcements using a text extraction system. The spider will add all found and relevant information to the ITTALKS knowledge base

\section{Conclusion}

Effective use of the vast quantity of information now available on the web necessitates semantic markup such as DAML. With the use of such a tool, we can enable the automated or machine-facilitated gathering and processing of much information that is currently 'lost' to us. ITTALKS, our system for automatic and intelligent notification of Information Technology talks, demonstrates the value of DAML in a variety of ways. DAML is used throughout the ITTALKS system, from basic knowledge representation, to inter-agent communication.

\section{References}

AeroText site, http://mds.external.lmco.com/Products_Services/aero/

Fabio Bellifemine, Agostino Poggi, and Giovanni Rimassa. Developing multi agent systems with a fipa-compliant agent framework. Software - Practice and Experience, (3), 2001.

Tim Berners-Lee and Mark Fischetti, Weaving the Web: The Original Design and Ultimate Destiny of the World Wide Web by its Inventor, Harper, San Francisco, 1999.

Tim Berners-Lee, James Hendler and Ora Lassila, The Semantic Web, Scientific American, May 2001.

Kurt D. Bollacker, Steve Lawrence, and C. Lee Giles. Citeseer: An autonomous web agent for automatic retrieval and identification of interesting publications. In Proceedings of the Second International Conference on Autonomous Agents (Agents '98), Minneapolis, 1998. ACM Press.

Sergey Brin and Lawrence Page, The Anatomy of a LargeScale Hypertextual Web Search Engine, Proceedings of the 7th International World Wide Web Conference, April 1998.

Harry Lik Chen, D. Chakraborty, L. Xu, Anupam Joshi, and Tim Finin. Service discovery in the future electronic market. In tim finin and Benjamin Grosof, editors, Proceedings of the AAAI Workshop on Knowledge Based Electronic Markets. AAAI Press, August 2000.

Harry Lik Chen, Anupam Joshi, Tim Finin, and D. Chakraborty. Dynamic service discovery for mobile 
computing: Intelligent agents meet jini in the aether. Baltzer Science Journal on Cluster Computing, 2001.

Lois C. Childs, AeroText - A Customizable Information Extraction System, unpublished technical report, Lockheed Martin, 2001.

Fabio Ciravegna "Learning to Tag for Information Extraction from Text" in Fabio Ciravegna, Roberto Basili, Robert Gaizauskas (eds.) ECAI Workshop on Machine Learning for Information Extraction, workshop held in conjunction with ECAI2000, Berlin, August 2000.

R. Scott Cost, Ye Chen, Tim Finin, Yannis Labrou and Yun Peng, Modeling agent conversations with colored petri nets, Working notes of the Workshop on Specifying and Implementing Conversation Policies, Autonomous Agents '99, Seattle, Washington, May, 1999.

R. Scott Cost, Tim Finin, Yannis Labrou, Xiaocheng Luan, Yun Peng, Ian Soboroff, James Mayfield and Akram Boughannam, "An Agent-based Infrastructure for Enterprise Integration", First International Symposium on Agent Systems and Applications, Palm Springs, October 36, 1999.

R. Scott Cost, Tim Finin, Yannis Labrou, Xiaocheng Luan, Yun Peng, Ian Soboroff, James Mayfield, and Akram Boughannam. Jackal: A java-based tool for agent development. In Jeremy Baxter and Chairs Brian Logan, editors, Working Notes of the Workshop on Tools for Developing Agents, AAAI '98, number WS-98-10 in AAAI Technical Reports, pages 73--82, Minneapolis, Minnesota, July 1998. AAAI, AAAI Press.

Neal Coulter et. al. Computing classification system 1998: Current status and future maintenance, report of the ccs update committee. Computing Reviews, January 1998.

DARPA Agent Markup Language site, http://www.daml.org.

Stefan Decker, Frank van Harmelen, Jeen Broekstra, Michael Erdmann, Dieter Fensel, Ian Horrocks, Michel Klein, Sergey Melnik: The Semantic Web - on the Roles of XML and RDF . In: IEEE Internet Computing. September/October 2000.

T. Eliassi-Rad \& J. Shavlik (2000). Instructable and Adaptive Web-Agents that Learn to Retrieve and Extract Information. Department of Computer Sciences, University of Wisconsin, Machine Learning Research Group Working Paper 2000-1.

D. Fensel et al.: OIL in a nutshell In: Knowledge Acquisition, Modeling, and Management, Proceedings of the European Knowledge Acquisition Conference (EKAW-
2000), R. Dieng et al. (eds.), Lecture Notes in Artificial Intelligence, LNAI, Springer-Verlag, October 2000.

Tim Finin, Yannis Labrou, and James Mayfield, KQML as an agent communication language, invited chapter in Jeff Bradshaw (Ed.), "Software Agents", MIT Press, Cambridge, (1997).

FIPA. FIPA 97 specification part 2: Agent communication language. Technical report, FIPA - Foundation for Intelligent Physical Agents, october 1997.

Benjamin Grosof and Yannis Labrou, An approach to using XML and a rule-based content language with an Agent Communication Language, IJCAI-99 Workshop on Agent Communication Languages, Stockholm, July 1999. Also available as IBM Research Report RC 21491. Revised version to appear as chapter in the book: Issues in Agent Communication, edited by Frank Dignum and Mark Greaves, Springer-Verlag, forthcoming.

Benjamin Grosof, Yannis Labrou and Hoi Chan, A Declarative Approach to Business Rules in Contracts: Courteous Logic Programs in XML, First ACM Conference on Electronic Commerce (EC-99), Denver, Nov 1999.

N. Guarino. Formal ontology and information systems. In N. Guarino, editor, Formal Ontology in Information Systems. IOS Press, 1998.

Jeff Heflin, James Hendler, Sean Luke, SHOE: A Prototype Language for the Semantic Web, Linköping Electronic Articles in Computer and Information Science, ISSN 1401-9841, Vol. 6 (2001).

James Hendler, Agents and the Semantic Web, IEEE Intelligent Systems, vol. 16, no. 2, Mar./Apr. 2001, pp. 3037.

James Hendler and Deborah McGuinness, The DARPA Agent Markup Language, IEEE Intelligent Systems, vol. 15, no. 6, Nov./Dec. 2000, pp. 72-73.

IBM Corporation, IBM CommonRules home page, http://www.research.ibm.com/rules/, which includes link to the download site http://alphaworks.ibm.com .

R. Kavasseri, T. Keating, M. Wittman, A. Joshi and S.

Weerawarana, "Web Intelligent Query - Disconnected Web Browsing with Collaborative Techniques", Proc. First IFCIS Conf. On Cooperative Information Systems (CoopIS96), Brussels, 1996.

Jackal site, http://jackal.cs.umbc.edu/.

Lalana Kagal, Harry Chen, Scott Cost, Timothy Finin, and Yun Peng. An infrastructure for distributed trust management. Submitted to Autonomous Agents Workshop 
on Norms and Institutions in Multiagent Systems, March 2001.

Lalana Kagal, Vlad Korolev, Harry Chen, Anupam Joshi, and Timothy Finin. Centaurus: A framework for intelligent services in a mobile environment. In Proceedings of the International Workshop on Smart Appliances and Wearable Computing (IWSAWC), April 2001.

Robert Kass and Tim Finin. General user modelling: A facility to support intelligent interaction. In J. Sullivan and S. Tyler, editors, Architectures for Intelligent Interfaces: Elements and Prototypes. Addison-Wesley, 1990.

Craig A. Knoblock, Kristina Lerman, Steven Minton, and Ion Muslea. Accurately and reliably extracting data from the web: A machine learning approach. Data Engineering Bulletin.

Ora Lassila. Web metadata: A matter of semantics. IEEE Internet Computing, 2(4):30-37, 1998.

Ora Lassila and Deborah McGuinness, The Role of FrameBased Representation on the Semantic Web. Linköping Electronic Articles in Computer and Information Science, ISSN 1401-9841, Vol. 6 (2001).

Ninghui Li, Joan Feigenbaum, and Benjamin Grosof, A Logic-based Knowledge Representation for Authorization with Delegation (Extended Abstract), Proc. 12th IEEE Computer Security Foundations Workshop, Mordano, Italy, June 1999. Extended version (with full proofs), by N. Li, B. Grosof, and J. Feigenbaum, available as IBM Research Report RC 21492.

N. Li and B. Grosof. A practically implementable and tractable delegation logic. In IEEE Symposium on Security and Privacy, May 2000.

MapQuest site, http://www.mapquest.com/.

James Mayfield, Paul McNamee and Christine Piatko, 'The JHU/APL HAIRCUT system at TREC-8.' In The Eighth

Text Retrieval Conference (TREC-8), E. M. Voorhees and D. K. Harman, eds., November 1999, pp. 445-452.

McCallum, Andrew Kachites. "Bow: A toolkit for statistical language modeling, text retrieval, classification and clustering.", http://www.cs.cmu.edu/ mccallum/bow. 1996.

Sheila A. McIlraith, Tran Cao Son, and Honglei Zeng, Semantic Web Services, IEEE Intelligent Systems, vol. 16, no. 2, Mar./Apr. 2001.

R. Neches, R. Fikes, T. Finin, T. Gruber, R. Patil, T. Senator and W. Swartout, 'Enabling Technology for
Knowledge Sharing", \{AI Magazine, v. 12, N. 3, pp 37-56, 1991.

RDF site, http:/www.w3c.org/RDF.

RDFS, http://www.w3.org/TR/rdf-schema/.

RSS, http://my.netscape.com/publish/formats/rss-spec-0.91

S. Staab, M. Erdmann, and A. Maedche, Ontologies in RDF(S), Linköping Electronic Articles in Computer and Information Science, ISSN 1401-9841, Vol. 6 (2001).

WML, http://www1.wapforum.org/tech/documents/SPECWML-19991104.pdf

XML site, http://www.w3c.org/XML.

W3C Working Group. W3c resource description framework (rdf). October 1998.

W3C Working Group. W3c resource description framework (rdf) model and syntax specification. February 1999.

W3C Working Group. W3c resource description framework (rdf) schema specification. March 1999. 